\title{
Amplified Striatal Responses to Near-Miss Outcomes in Pathological Gamblers
}

\author{
Guillaume Sescousse $^{*, 1,2}$, Lieneke K Janssen ${ }^{1,2}$, Mahur M Hashemi', Monique HM Timmer ${ }^{1,3}$, \\ Dirk EM Geurts ${ }^{1,2}$, Niels P ter Huurne ${ }^{1,4}$, Luke Clark ${ }^{5}$ and Roshan Cools ${ }^{1,2}$ \\ 'Donders Institute for Brain, Cognition and Behaviour, Radboud University, Nijmegen, The Netherlands; '2Department of Psychiatry, Radboud \\ University Medical Centre, Nijmegen, The Netherlands; ${ }^{3}$ Department of Neurology, Radboud University Medical Centre, Nijmegen, \\ The Netherlands; ${ }^{4}$ Karakter Child and Adolescent Psychiatry University Centre, Nijmegen, The Netherlands; ${ }^{5}$ Department of Psychology, Centre for \\ Gambling Research at UBC, University of British Columbia, Vancouver, British Columbia, Canada
}

Near-misses in gambling games are losing events that come close to a win. Near-misses were previously shown to recruit reward-related brain regions including the ventral striatum, and to invigorate gambling behavior, supposedly by fostering an illusion of control. Given that pathological gamblers are particularly vulnerable to such cognitive illusions, their persistent gambling behavior might result from an amplified striatal sensitivity to near-misses. In addition, animal studies have shown that behavioral responses to near-miss-like events are sensitive to dopamine, but this dopaminergic influence has not been tested in humans. To investigate these hypotheses, we recruited 22 pathological gamblers and 22 healthy controls who played a slot machine task delivering wins, near-misses and full-misses, inside an $\mathrm{fMRI}$ scanner. Each participant played the task twice, once under placebo and once under a dopamine D2 receptor antagonist (sulpiride $400 \mathrm{mg}$ ), in a doubleblind, counter-balanced design. Participants were asked about their motivation to continue gambling throughout the task. Across all participants, near-misses elicited higher motivation to continue gambling and increased striatal responses compared with full-misses. Crucially, pathological gamblers showed amplified striatal responses to near-misses compared with controls. These group differences were not observed following win outcomes. In contrast to our hypothesis, sulpiride did not induce any reliable modulation of brain responses to near-misses. Together, our results demonstrate that pathological gamblers have amplified brain responses to near-misses, which likely contribute to their persistent gambling behavior. However, there is no evidence that these responses are influenced by dopamine. These results have implications for treatment and gambling regulation.

Neuropsychopharmacology (2016) 4I, 26I4-2623; doi: I0.1038/npp.2016.43; published online 27 April 2016

\section{INTRODUCTION}

Pathological gambling is a behavioral addiction with dramatic consequences including bankruptcy, unemployment, and relationship problems. The prevalence among college students has risen sharply in the past 10 years (Nowak and Aloe, 2014), while the rate of attempted suicide, approaching $20 \%$, is higher than for any other addictive disorder (Bischof et al, 2015). Central to pathological gambling is the loss of control over gambling behavior and the inability to stop playing. This compulsive behavior is postulated to be fueled by cognitive distortions regarding the game structure. One example is the misappraisal of nearmiss events-losing outcomes that come close to a winwhich invigorate ongoing gambling behavior, conceivably by

*Correspondence: Dr G Sescousse, Donders Centre for Cognitive Neuroimaging, Donders Institute for Brain, Cognition and Behaviour, Radboud University, PO Box 9101, Nijmegen $6500 \mathrm{HB}$, The Netherlands, Tel: +3| 2436 |06|8, Fax: +3| 2436 I0989, E-mail: g.sescousse@donders.ru.nl

Received 18 December 2015; revised 17 February 2016; accepted 18 March 2016; accepted article preview online 23 March 2016 fostering an illusion of control (Clark, 2010). Although the behavioral vulnerability of pathological gamblers to such cognitive distortions is well established (Michalczuk et al, 2011), the underlying neural and neurochemical mechanisms remain elusive. We aimed to address this question using a combination of fMRI and pharmacological manipulation.

Near-miss events are a hallmark of many gambling games and are particularly frequent in slot machines, which are recognized as one of the most addictive forms of gambling (MacLaren, 2015). In healthy individuals near-misses promote extended play (Côté et al, 2003; Kassinove and Schare, 2001) and enhance gambling motivation, especially in those with strong trait illusions of control (Billieux et al, 2012). In neuroimaging studies, near-misses recruit similar brain regions to wins, despite their objective loss status. Notably, near-misses elicit stronger activity than similarly non-winning 'full-miss' events in the ventral striatum, a core node of the brain reward circuitry (Chase and Clark, 2010; Clark et al, 2009; Worhunsky et al, 2014). In this context, an amplified striatal response to near-misses might be a plausible neurobiological mechanism contributing to pathological gambling. However, previous fMRI 
studies investigating this question have led to inconsistent results. Initial studies have found increased brain responses to near-misses in problem $v s$ non-problem gamblers (Habib and Dixon, 2010) as well as a positive correlation with symptom severity (Chase and Clark, 2010). However, other studies reported decreased striatal responses to near-misses in pathological gamblers compared with healthy controls (Worhunsky et al, 2014) as well as in healthy controls with high impulsivity, a trait that is commonly associated with pathological gambling (Shao et al, 2013).

One psychological account of near-misses postulates that these events operate by inducing inappropriate reinforcement learning signals (Clark, 2010 and Clark et al, 2013 for alternative hypotheses; but see Shao et al, 2013). Near-misses are proximal to a win, and accordingly, they may be wrongly interpreted as a sign of skill acquisition (illusion of control), thereby enhancing gambling motivation. This account is in line with observed neural signaling in the ventral striatum, which is often implicated in reinforcement learning (Delgado, 2007). One key implication of this hypothesis is that near-miss responses should be sensitive to dopamine. Dopamine has a central role in reinforcement learning, and modulates both brain and behavioral responses to learning feedback (Cools et al, 2006; Maia and Frank, 2011; Pessiglione et al, 2006). In addition, animal studies have shown that increasing dopamine transmission with amphetamine or the D2-like receptor agonist quinpirole enhances an analog of the near-miss effect in rats (Cocker et al, 2014; Winstanley et al, 2011). Our study aimed to test this hypothesis in humans.

Pathological gambling has been associated with dopaminergic dysfunction, and more specifically with a hyperdopaminergic state in the striatum. In particular, PET studies have shown enhanced amphetamine- and gambling-induced dopamine release in the striatum of individuals with pathological gambling symptoms, as compared with healthy controls (Boileau et al, 2014; Steeves et al, 2009). Dopamine D3 receptor binding in the substantia nigra was also shown to be positively correlated with gambling severity (Boileau et al, 2013). These results are in line with the observation that high dopamine states are associated with increased risktaking in healthy humans (Oswald et al, 2015) and that dopamine D2/D3 receptor agonists increase risk-taking in both humans and animals (Morgado et al, 2014; Riba et al, 2008; St Onge and Floresco, 2009). On the basis of these results, we hypothesized that altering dopamine transmission with a dopamine D2/D3 receptor antagonist would alter striatal responses to near-misses in pathological gamblers.

To address the above questions, we set up a pharmacofMRI study in which pathological gamblers and healthy controls played a slot machine task that delivered wins, nearmisses, and full-misses. The task was similar to that used in previous studies showing robust striatal responses to nearmisses (Chase and Clark, 2010; Clark et al, 2009). We manipulated dopamine in a placebo-controlled, withinsubjects manner using the selective D2/D3 receptor antagonist sulpiride. Sulpiride has previously been shown to modulate learning (Eisenegger et al, 2014; Mehta et al, 2008; van der Schaaf et al, 2014) as well as brain and behavioral responses to reward and punishment (Becker et al, 2013; Jocham et al, 2014; McCabe et al, 2011). Our primary interest was in the comparison of near-miss and full-miss events. We expected to see an enhanced striatal response to near-misses compared with full-misses in both groups, and that this response would be amplified in the pathological gamblers compared with controls. Given the contribution of prefrontal regions to gambling-related cognitive distortions (Xue et al, 2013, 2012), we further explored their influence on the striatum using functional connectivity.

\section{MATERIALS AND METHODS}

\section{Subjects}

Twenty-two male pathological gamblers and twenty-two healthy controls participated in the study, following an indepth structured psychiatric interview administered by a medical doctor (MINI Plus; Sheehan et al, 1998). All subjects provided written informed consent, which was approved by the regional research ethics committee (Commissie Mensgebonden Onderzoek, region Arnhem-Nijmegen).

Pathological gamblers were recruited through advertisement $(N=18)$ and addiction clinics $(N=4)$. None of the gamblers was in treatment at the time of testing, except for two of them enrolled in cognitive behavioral therapy (one for cannabis dependence-see below-and another one who was just starting a cognitive behavioral therapy for his gambling problems). Controls were recruited through advertisement. All gamblers, with the exception of one, qualified as pathological gamblers ( $\geqslant 5$ DSM-IV criteria for pathological gambling). One gambler qualified as problem gambler as he met only four DSM-IV criteria. The severity of gambling symptoms was assessed using the South Oaks Gambling Screen (SOGS; Lesieur and Blume, 1987). All gamblers had a minimum SOGS score of 6 (range $=6-18$ ), whereas controls, with the exception of two subjects, had a SOGS score of 0 (range $=0-2$ ).

The two groups were matched for age, net income, body mass index, and verbal IQ (Table 1). Subjects were excluded if they consumed more than four alcoholic beverages daily; were using psychotropic medication; had a lifetime history of schizophrenia, bipolar disorder, attention deficit hyperactivity disorder, autism, eating disorder, anxiety disorder, or obsessive compulsive disorder; or had a past 6 months history of major depressive episode. Given the high comorbidity between pathological gambling and other psychiatric disorders (Lorains et al, 2011), gamblers with the following co-morbidities were included: current cannabis dependence $(N=1)$; past cannabis dependence ( $>5$ months; $N=1)$; lifetime history of dysthymia $(N=1)$; and remitted post-traumatic stress disorder (remitted $>4$ years; $N=2$ ). In addition, three gamblers used cannabis weekly in the past 6 months, but did not meet the DSM-IV criteria for abuse/ dependence. The control subjects did not have any history of substance abuse or dependence. A number of self-report questionnaires were further used to characterize the subjects (Table 1 and Supplementary information).

\section{Procedure}

Subjects were tested once after receiving an oral dose of sulpiride (Dogmatil, $400 \mathrm{mg}$ ), and once after a placebo. The order of administration was randomized according to a 
Table I Demographic and Clinical Characteristics of Pathological Gamblers and Healthy Control Subjects

\begin{tabular}{|c|c|c|c|}
\hline & Healthy controls $(n=22)$ & Pathological gamblers $(n=22)$ & Group comparison $(p)$ \\
\hline Age & $32.2(11.1)$ & $35.7(8.8)$ & 0.26 \\
\hline Monthly income $(€)$ & $17 \mid 5.9(\mid 102.7)$ & | $727.3(865.7)$ & 0.97 \\
\hline Body mass index & $23.1(3.2)$ & $23.8(2.2)$ & 0.44 \\
\hline Fagerström test for nicotine dependence & $0.6(1.4)$ & $2.7(2.8)$ & 0.003 \\
\hline Number of current smokers & 10 & 14 & 0.23 \\
\hline Hospital Anxiety and Depression (anxiety subscale) & $2.6(2.8)$ & $5.2(3.4)$ & 0.008 \\
\hline Hospital Anxiety and Depression (depression subscale) & $1.5(2.3)$ & $5.0(4.5)$ & 0.003 \\
\hline National Adult Reading Test—Verbal IQ score & I05.2( (10.3) & $99.6(12.3)$ & 0.11 \\
\hline South Oaks Gambling Screen & $0.2(0.5)$ & $12.4(3.9)$ & $<0.001$ \\
\hline Gambling Beliefs Questionnaire (GBQ) _ Overall & I23.2( (16.8) & $82.0(23.1)$ & $<0.001$ \\
\hline
\end{tabular}

All values are mean (SD). Groups were compared using two-sample t-tests, except for the number of current smokers where a Chi-square test was used.

double-blind, cross-over design (placebo-sulpiride: 10 controls, 11 gamblers; sulpiride-placebo: 12 controls, 11 gamblers). The test sessions were separated by at least 1 week. Background neuropsychological functioning, physiological measures and subjective mood were measured at several time points during the protocol (Supplementary information, Supplementary Tables S1-S3). The slot machine task (of primary interest here) was part of a larger protocol investigating the sensitivity to gains and losses within the context of a reversal learning task (Janssen et al, 2015), a mixed gamble task, and a probability discounting task. The slot machine task was performed approximately $1 \mathrm{~h}$ 45 min after drug intake, thus coinciding with maximal effects of sulpiride (Mehta et al, 2003).

\section{Task}

We employed a two-reel slot machine task, similar to the one used in Clark et al (2009). The task was programmed using Neurobs Presentation (version 14.1), using sounds and 3D graphics to make the task as engaging as possible. Each trial consisted of four phases: choice, anticipation, outcome, and rating (Figure 1). In the choice phase, subjects had a maximum of $5 \mathrm{~s}$ to select one of six playing symbols on the left reel of the slot machine. Note that in contrast to Clark et al (2009), we did not include trials in which the playing symbol was selected by the computer, as this condition was not associated with the invigorating effect of near-misses. Following selection, the right reel spun for a variable duration anticipation phase (3.36-6.95 s) and decelerated to a standstill. In the outcome phase $(3 \mathrm{~s})$, if the right reel stopped on the same symbol as the one selected on the left reel, subjects won $5 €$ accompanied by a cash register sound. In all other cases, subjects did not win any money and heard a buzzer sound. Near-miss outcomes were defined as those where the right reel stopped one position away from the selected symbol. Full-miss outcomes were defined as those where the right reel stopped two or three positions away from the selected symbol. Finally, in the rating phase, subjects answered the question 'How much do you want to continue to play?' using a continuous scale ranging from 'Not at all' to 'A lot'. Each trial ended with an intertrial interval of variable length $(2-5 \mathrm{~s})$.

The task was divided into three runs of 30 trials each. Outcomes were pseudorandomized to ensure a fair distribution of wins $(1 / 6,15$ in total), near-misses $(2 / 6,15$ below the payline, 15 above the payline), and full-misses $(3 / 6,45$ in total). The sequence of outcomes was fixed to ensure a roughly equal distribution of the three types of outcomes across the three runs. This sequence was different for the 2 days, and the order was counter-balanced across drug sessions. Subjects were given oral instructions and practiced the task both before entering the scanner (12 trials) and within the scanner ( 8 trials). Subjects received a fixed amount of $50 €$ for their participation, plus a bonus corresponding to the mean earnings across the three runs, which amounted to $25 €$ for all subjects due to the pseudorandomized sequence of outcomes.

\section{fMRI Data Acquisition}

Imaging was conducted on a Siemens TIM Trio 3T scanner, using a 32-channel head coil. Whole brain $\mathrm{T} 2{ }^{*}$-weighted BOLD fMRI data were acquired using multi-echo echoplanar imaging (Supplementary Information).

\section{fMRI Data Analysis}

Preprocessing and statistical analyses of fMRI data were performed with SPM8. Preprocessing steps involved realignment, slice-timing correction, normalization to the MNI space and spatial smoothing (Supplementary Information).

We used the summary statistics approach for the statistical analysis, in which the data from each participant (including both drug sessions) were analyzed separately in a first-level analysis based on the general linear model (GLM), and then combined in a second-level, random effects analysis. Our first-level GLM incorporated separate regressors for the 


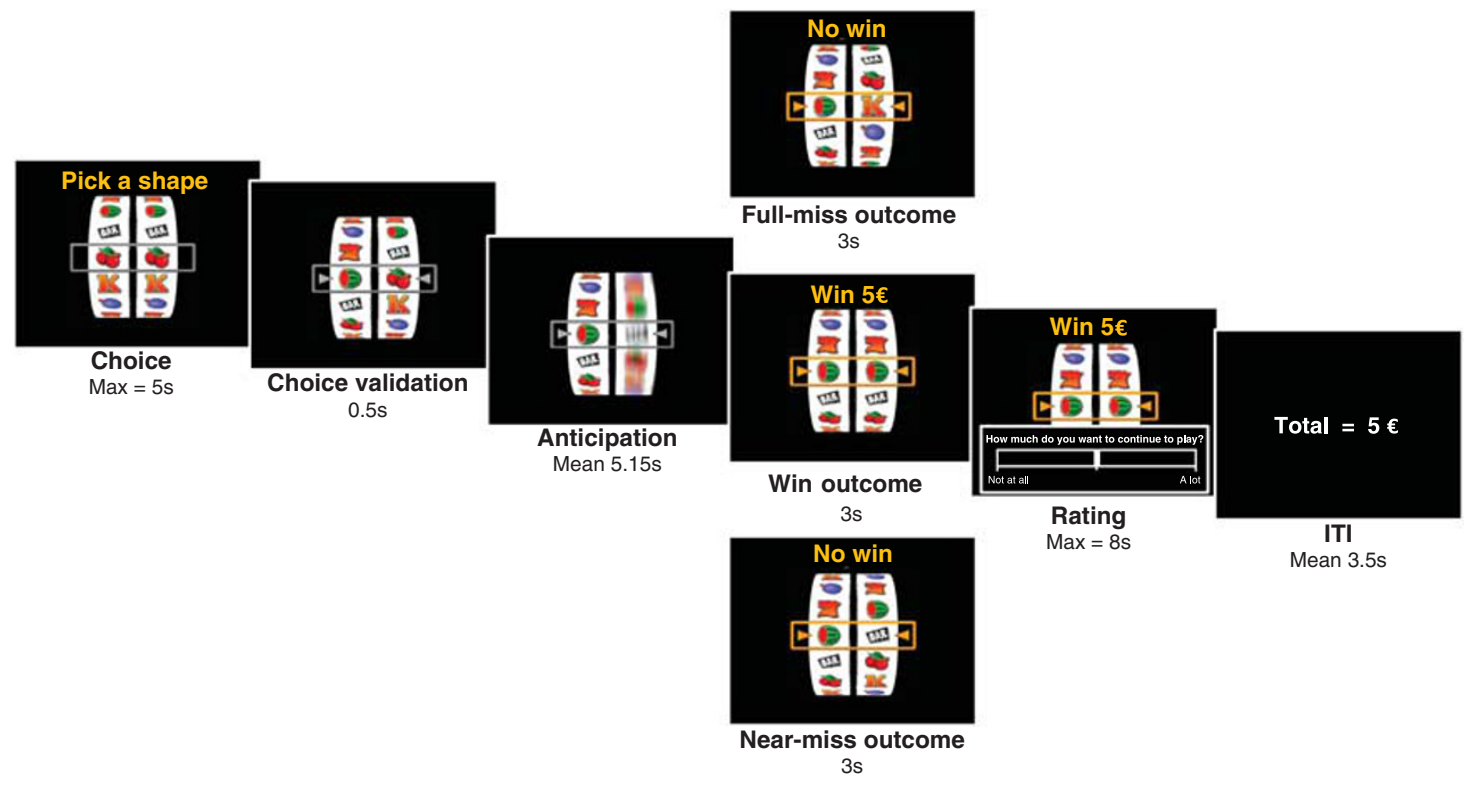

Figure I Slot machine task. In the initial choice phase, subjects had a maximum time of $5 \mathrm{~s}$ to select one of six playing symbols on the left reel of the slot machine. They could move the reel downward or upward with buttons I and 2 (index and ring finger), and validate their choice with button 3 (middle finger). Following choice validation, the anticipation phase began, in which the right reel spun for a variable duration (3.36-6.95 s) and decelerated to a standstill. The variability of the spinning duration was introduced to enhance the unpredictability of the outcome. The outcome phase started when the reel stopped and the payline turned orange simultaneously. If the right reel stopped on the same symbol as the one selected on the left reel, then subjects won $5 €$ and heard a cash register sound. If the right reel stopped one position away (near-miss) or two/three positions away from the payline (full-miss), then subjects won nothing and heard a buzzer sound. Note that near-misses and full-misses were not signaled differently to the subjects. Finally, after each outcome, subjects were asked to rate their motivation to continue gambling, on a continuous scale from 'not at all' to 'A lot'. They could move a cursor (initially positioned in the middle) left or right with buttons I and 2, and validate their choice with button 3. These ratings were coded into numeric values ranging from 0 to 10 with an increment of 0.I. Consecutive trials were separated with a variable intertrial interval (ITI). If symbol selection or the rating was not completed within the maximum time allocated, then a 'Too late!' message appeared for I $\mathrm{s}$ and the trial continued.

choice phase, the anticipation phase, the outcome phase, and the rating phase. The choice phase was modeled as a boxcar with a duration equal to the time between the appearance of the reel and the choice validation by the subject. The anticipation phase was modeled as a boxcar with a duration equal to the spinning time of the right reel. The three possible outcomes (win, near-miss, and full-miss) were modeled as three separate events. Finally, the rating phase was modeled as a boxcar with a duration equal to the time between the appearance of the scale and the rating validation by the subject. All these regressors were subsequently convolved with a canonical hemodynamic response function. Twelve motion parameters, including the six realignment parameters and their first derivatives, were included in our GLM. In addition, the time series of the mean signal from the white matter, cerebral spinal fluid, and out-of-brain segments were added as nuisance parameters modeling global intensity changes (Verhagen et al, 2008). Data were also high-pass filtered (128s) to remove low-frequency signals; and an AR(1) model was applied to adjust for serial correlations in the data.

At the second level, we first examined the contrast (nearmiss $>$ full-miss) across drug sessions. This was done within each group separately and between groups. Then, we examined the main effect of drug on the contrast (nearmiss $>$ full-miss) across groups, as well as within each group separately. Finally, we examined the interaction of drug and group on the contrast (near-miss $>$ full-miss). Comparisons within and across groups were performed using one-sample $t$-tests; and comparisons between groups were performed using two-sample $t$-tests. We used the same procedure to examine the response to wins compared with non-wins, using the contrast (win $>$ near-miss and full-miss). Results were thresholded with a voxel-wise $p<0.05$ family-wise error (FWE) corrected for multiple comparisons across the wholebrain $\left(p_{\mathrm{FWE}}\right.$ whole-brain $\left.<0.05\right)$. On the basis of our a priori hypothesis about the role of the ventral striatum in pathological gambling as well as near-misses, we further applied this correction to a small volume corresponding to this region $\left(p_{\text {FWE }}\right.$ small volume $\left.<0.05\right)$. This volume was defined as the union of two $10-\mathrm{mm}$ spheres centered around the striatal peak voxels reported in a previous study for the same contrast of near-misses vs full-misses (Clark et al, 2009).

To illustrate our whole-brain results, we extracted the percent signal change from several functional clusters using the rfxplot toolbox (Gläscher, 2009). To investigate potential changes of fronto-striatal coupling as a function of outcome and group, we examined functional connectivity using a generalized PsychoPhysiological Interaction (gPPI, Supplementary Information).

\section{RESULTS}

\section{Behavioral Results}

Motivation ratings were first analyzed using a four-way ANOVA with Outcome (win/near-miss/full-miss) and Drug (sulpiride/placebo) as within-subject factors, and 

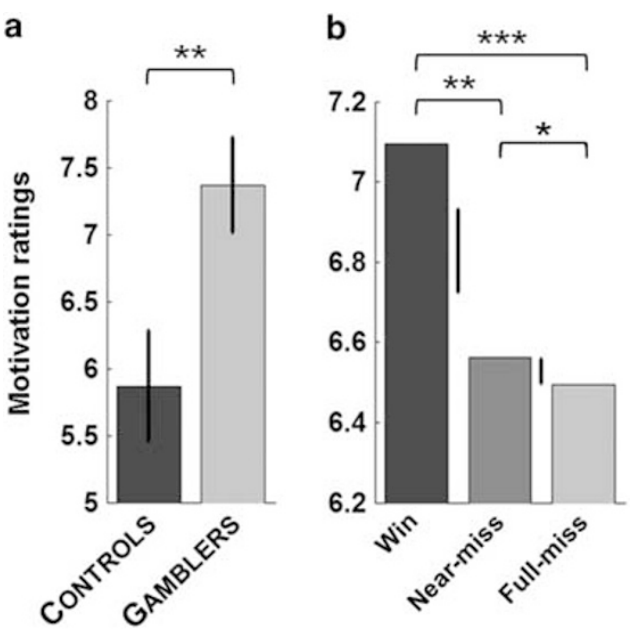

Figure 2 Motivation ratings on the slot machine task. (a) There was a main effect of group on motivation ratings to continue gambling, indicating a higher motivation in gamblers compared with controls. Error bars indicate standard error of the mean (SEM). (b) There was also a main effect of outcome on motivation ratings, with higher ratings for win than near-miss and full-miss outcomes, and higher ratings for near-miss than full-miss outcomes (across groups and drug sessions). Error bars indicate standard error of the difference between conditions. Asterisks denote significance of planned contrasts: $* * * * * 0.001$, $* * * 0.01$, $* 2<0.05$.

Group (controls/gamblers) and Drug Order (placebo-sulpiride/sulpiride-placebo) as between-subject factors (Figure 2). The motivation to continue gambling was higher in the pathological gamblers than in the healthy controls, as indicated by a main effect of Group $(F(1,40)=7.58, p=0.009)$. Motivation was also modulated by the type of preceding outcome, as indicated by a strong main effect of Outcome $(\mathrm{F}(2,80)=27.25, p<0.001)$. As expected, wins elicited higher motivation than near-misses and full-misses $(\mathrm{F}(1,40)=28.57$, $p<0.001$ ), and in line with previous studies (Clark et al, $2009,2013)$, near-misses elicited higher motivation than fullmisses $(\mathrm{F}(1,40)=4.83, p=0.034)$. The effect of Outcome on motivation did not differ between groups, as revealed by a nonsignificant Group $\times$ Outcome interaction $(p=0.76)$. There was no main effect of Drug or any Drug $\times$ Group or Drug $\times$ Outcome interactions (all $p>0.19$ ). Drug Order did not interact with the Drug factor $(p=0.29)$, suggesting no reliable session effects.

We further investigated changes in motivation ratings over time, using a four-way ANOVA with Outcome, Drug, and Run $(1 / 2 / 3)$ as within-subject factors, and Group as a between-subject factor. The main effect of Run was significant $(\mathrm{F}(2,84)=12.43, p<0.001)$, reflecting a decline of motivation to continue gambling over time. However, there were no significant Run $\times$ Group or Run $\times$ Outcome $\times$ Group interactions (all $p>0.37$ ), suggesting that this decline was not significantly different between groups nor did it vary differently between groups as a function of outcome.

In the pathological gamblers, higher levels of distortions (reflected by lower GBQ scores) predicted higher motivational ratings after all three outcomes (all $r \leqslant-0.44$, $p<0.05)$. These correlations were not present in controls (all $p>0.29$ ).

\section{fMRI Results}

All analyses referred to in this section can be accessed at http:// neurovault.org/collections/1186/. Replicating previous results (Clark et al, 2009), we found that across drug sessions nearmisses elicited stronger BOLD signal than full-misses in the bilateral ventral striatum, in both controls $(x, y, z=-10,8,0$, $T=9.40 ; 10,13,0, T=8.00, p_{\text {FWE }}$ whole-brain $\left.<0.005\right)$ and pathological gamblers $(x, y, z=-10,10,0, T=13.61 ; 12,10,2$, $T=13.33, p_{\text {FWE }}$ whole-brain $<0.001$ ) (Figure $\left.3 a\right)$. Similar results were observed in the bilateral anterior insula (Supplementary Tables S4 and S5). In line with our hypothesis, striatal responses to near-misses (compared with full-misses) were stronger in pathological gamblers than in controls $(x, y, z=-10$, $10,0, T=3.88 ; 12,10,2, T=3.97, p_{\text {FWE small volume }}<0.05$ ) (Figure $3 \mathrm{~b}$ ). Whole-brain analyses did not reveal any other significant group differences (Supplementary Tables S6 and S7). Extraction of percent signal in the ventral striatum change further revealed that this Outcome $\times$ Group interaction was driven by an amplified response to near-misses in pathological gamblers compared with controls, while the response to full-misses was comparable across groups. Additional sensitivity analyses showed that the above group difference was unlikely to be driven by potentially confounding variables such as smoking, anxiety, or depression (Supplementary Information). We also examined whether brain responses to near-misses were modulated by GBQ scores or motivation ratings across participants in each group. These analyses did not reveal any significant correlations, either at the whole-brain level or in small striatal and insular regions of interest derived from the above analyses.

We then tested whether the enhanced striatal response in gamblers extended to win outcomes, using the contrast between wins and all non-win outcomes (ie, near-misses and full-misses). As expected, this contrast revealed strong signal change in each group throughout the reward system, particularly in the ventral striatum (Supplementary Figure S1A, Supplementary Tables S8 and S9). However, there were no significant differences between controls and gamblers, either at the whole-brain level or within our striatal volume of interest (Supplementary Figure S1B, Supplementary Tables S10 and S11).

We further investigated whether the enhanced striatal response to near-misses in gamblers was accompanied by a differential fronto-striatal connectivity, given the role of prefrontal regions in cognitive distortions. Using the striatal voxels from the group difference map as a seed, we examined gPPI connectivity with the rest of the brain as a function of Outcome (near-misses $v s$ full-misses) and Group. There were no supra-threshold effects according to our stringent wholebrain voxel-wise statistical thresholding procedure, although for completeness we describe in the Supplementary Information an interesting gPPI in the left lateral prefrontal cortex at a whole-brain cluster-level corrected threshold (Supplementary Figure S2).

Finally, we investigated the effect of our pharmacological manipulation on the brain responses to near-misses compared with full-misses. We did not observe any drug effects across groups, either at the whole brain level or within our striatal volume of interest. Similarly, we did not observe any drug effects in either group examined separately (Supplementary Figure S3). This absence of drug effect was 
a

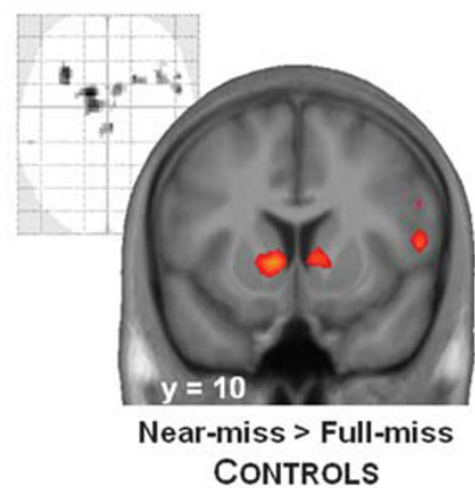

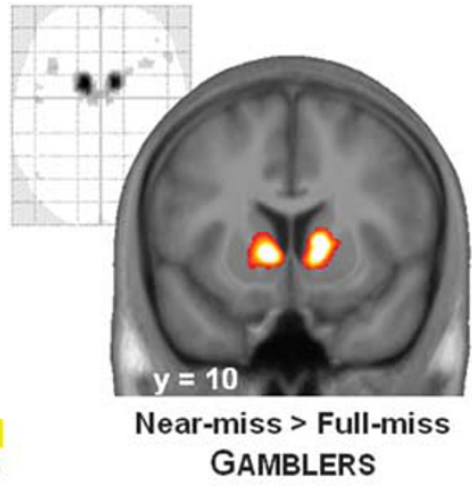

T

6.75

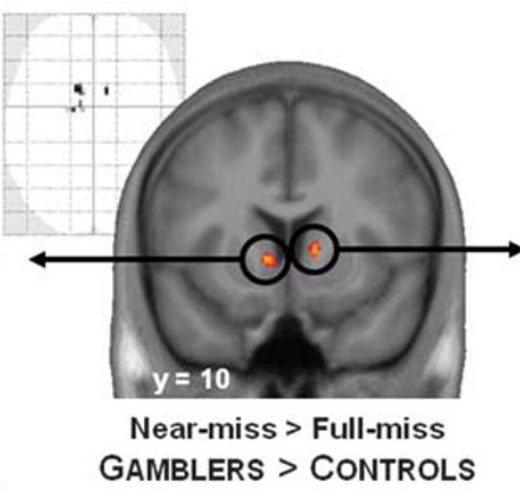

GAMBLERS > CONTROLS

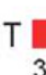

T

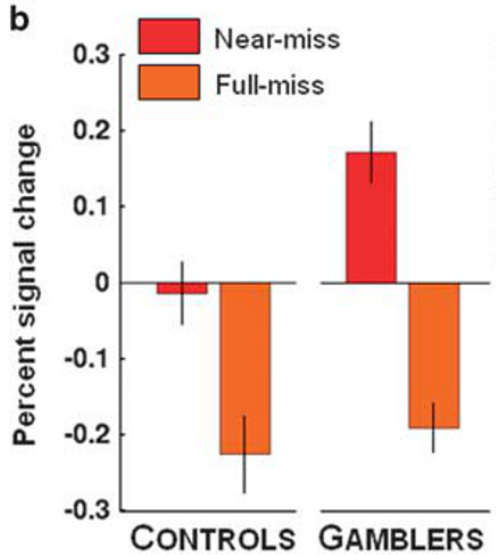

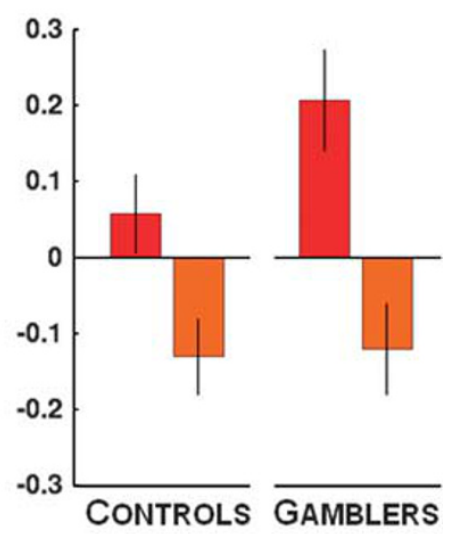

Figure 3 Amplified striatal response to near-misses in gamblers. (a) T-map and glass brain showing increased striatal response to near-misses compared with full-misses in controls and pathological gamblers. Activations are overlaid on an average anatomical scan of all subjects (display threshold: $p<0.05 \mathrm{FWE}$ wholebrain corrected). (b) T-map and glass brain showing amplified striatal response to near-misses (as compared with full-misses) in gamblers compared with controls. Activations are overlaid on an average anatomical scan of all subjects (display threshold: $p<0.001$ uncorrected, $k=10$ ). Note that peak activations in the bilateral striatum survive an FWE-corrected threshold of $p<0.05$ within 10-mm spheres centered on peak coordinates extracted from a previous independent study (Clark et al, 2009) (left striatum: $x, y, z=-8,4,-2$; right striatum: $x, y, z=12,2,-2$ ). The plots of mean percent signal change (extracted from left and right striatal voxels significant at $p<0.001$ uncorrected, $k=10$ ) further illustrate that the group difference is mostly driven by an increased response to near-misses in gamblers, rather than a decreased response to full-misses. Error bars indicate SEM.

further illustrated by the extraction of percent signal change in the striatum, which showed a very similar pattern of activation in the sulpiride and placebo conditions (Figure 4). The striatal responses to near-misses were actually strongly correlated between drug sessions across all participants $(r=0.51, p<0.001)$, suggesting that it represents a relatively stable trait. Finally, direct group comparisons did not reveal any differential effect of sulpiride between gamblers and controls, either at the whole brain level or within our striatal volume of interest. We also checked the sulpiride effect on the contrast between win and non-win outcomes, and similarly did not observe any reliable modulation of winrelated activity.

\section{DISCUSSION}

The present study demonstrates that ventral striatal responses to near-misses are amplified in pathological gamblers compared with healthy controls. Such a group difference was not present following win outcomes. In addition, contrary to our hypothesis, we did not observe any modulation of brain responses to near-misses by sulpiride.

Our results advance earlier findings that showed qualitatively enhanced responses to near-misses in the midbrain of problem gamblers (Chase and Clark, 2010; Habib and Dixon, 2010). The present study extends these previous observations to the striatum and establishes, for the first time, a quantitative difference in near-miss responses between clinically characterized pathological gamblers and healthy controls. The recruitment of the reward system by nearmisses is postulated as a mechanism that underlies the invigorating effect of these events on gambling behavior (Clark et al, 2009). Several hypotheses have been formulated regarding the underlying psychological mechanism. The most popular hypothesis builds on the prominent role of the ventral striatum in reinforcement learning, and posits that enhanced activity in the striatum would reflect aberrant learning about skill acquisition (Billieux et al, 2012). Given that such skills are irrelevant in a context where outcomes depend purely on chance, this aberrant learning contributes to an illusion of control. This idea is consistent with the fact that pathological gamblers are known to maintain strong 


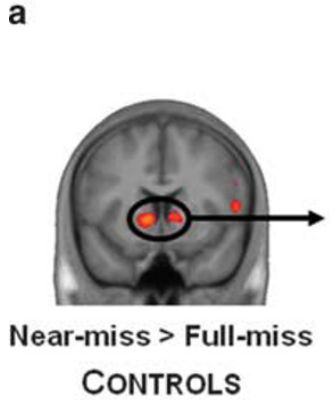

CONTROLS

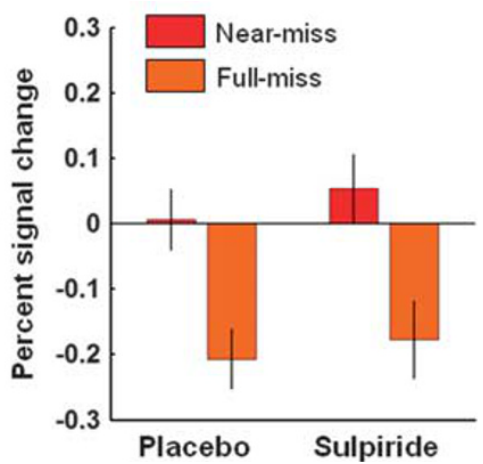

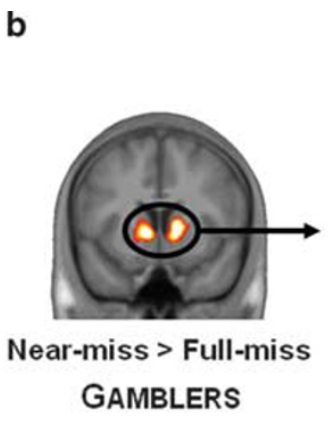

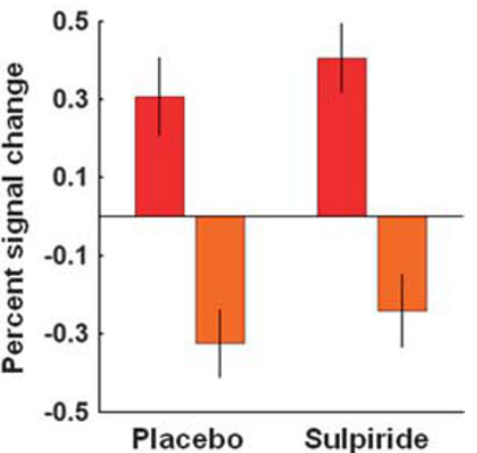

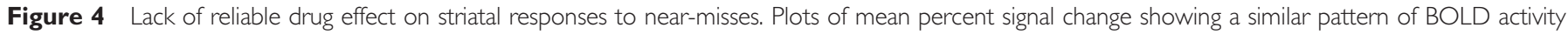

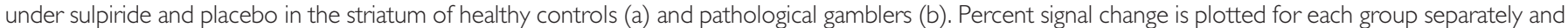

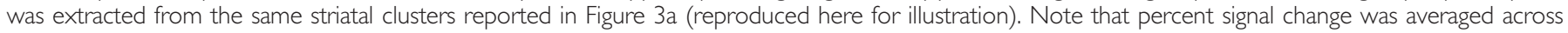
hemisphere for simplicity, but results are similar in both hemispheres. Error bars indicate SEM.

gambling-related cognitive distortions, as illustrated, eg, by low scores on the Gambling Beliefs Questionnaire in our study (Table 1). It is further in line with the negative correlation observed between these scores and the motivation to continue gambling following near-misses in pathological gamblers (although this correlation was also observed following wins and full-misses).

An alternative account of near-misses suggests a link with the aversive emotional states triggered by missing the jackpot, such as frustration (Dixon et al, 2011). In line with this idea, near-misses are often rated as less pleasant than full-misses and elicit larger skin conductance responses (Clark et al, 2009; Dixon et al, 2013; Stange et al, 2015). Accordingly, it is possible that the enhanced striatal response to near-misses seen here reflects enhanced negative arousal and frustration (see eg, Shao et al, 2013).

Finally, some authors have suggested that near-misses boost gambling motivation in part by generating 'upward' counter-factual thoughts, where players mentally simulate the missed win (Clark et al, 2013; Wu et al, 2015). This hypothesis predicts different reactions to near-misses before $v s$ after the payline, as counter-factual thoughts differ in these two situations. In line with this idea, Clark et al (2013) have shown that near-misses before the payline are more motivating while near-miss after the payline is more unpleasant and lead to higher skin conductance responses. However, supplementary analyses (not shown here) did not reveal any differences between near-misses before and after the payline, and our results are thus unable to support this counter-factual account.

Our results also revealed robust responses to near-misses in the bilateral anterior insula, consistent with previous reports (Chase and Clark, 2010; Clark et al, 2009). In particular, a lesion study in humans showed that insula damage abolishes the boost in motivation induced by nearmisses (Clark et al, 2014). Given the role of the insula in interoception, it has been hypothesized that the effect of near-misses might be partly mediated by an abnormal representation of gambling-related bodily states. Although our results are not in contradiction with this hypothesis, the absence of group difference in the insula (even at a very liberal threshold of $p<0.05$ uncorrected) suggests that the enhanced gambling-related cognitive distortions observed in pathological gamblers might not be subserved by abnormal processing of bodily states, but rather by inappropriate reinforcement learning as previously suggested.

As expected, motivation ratings to continue gambling were higher following near-misses than full-misses, and were overall higher in pathological gamblers than in controls. However, there was no group-by-outcome interaction, and thus no evidence of an enhanced invigorating effect of nearmisses in gamblers compared with controls. We suspect that this might be due to the limited sensitivity of our subjective rating procedure for measuring gambling motivation: the repetition of ratings on every trial, combined with the lack of behavioral relevance of these ratings for the participants, might have made the rating procedure boring and thus less reliable for some participants. In the future, more sensitive measures of gambling motivation such as persistent play (Billieux et al, 2012; Clark et al, 2013) or perhaps salivation (Wadhwa and Kim, 2015) might be used to reveal the boosting effect of near-misses on motivation in pathological gamblers.

Interestingly, our results did not reveal any group differences in reward-related brain regions following win outcomes. This negative result is unlikely to reflect a lack of statistical power, given that we observed a clear group difference in the contrast between near-misses and fullmisses. This observation bears relevance in the context of the recent debate questioning whether or not pathological gamblers have a hypo-active reward system (Leyton and Vezina, 2012). Whereas some studies have reported blunted brain responses to monetary rewards in pathological gamblers (Balodis et al, 2012; de Ruiter et al, 2009; Reuter et al, 2005), others have reported normal to exaggerated responses (Hewig et al, 2010; Oberg et al, 2011; Sescousse et al, 2013). Importantly, we employed an ecologically valid slot machine task with relatively high amounts of money that were delivered for real, raising confidence that our observations do not reflect a lack of behavioral relevance as has been suggested for previous studies (Leyton and Vezina, 2012, 2013). In such a realistic context, our findings thus suggest that the sensitivity of pathological gamblers to gains might not be compromised, but that instead their sensitivity to certain losing events, ie, near-misses, might be distorted. 
Finally, in contrast to our hypothesis, sulpiride did not induce any detectable modulation of behavioral or brain responses to near-misses. This is consistent with a previous report showing no effect of haloperidol on near-miss responses in recreational gamblers, although in that study it was unclear whether haloperidol was functionally active (Porchet et al, 2013). In the present case, the negative results are unlikely to result from a lack of central action of sulpiride, since our drug manipulation showed clear effects in several other tasks within the same protocol (see eg, Janssen et al, 2015). One possibility is that sulpiride might produce a variable effect across participants, as a result of inter-individual differences in endogenous dopamine. Indeed, previous studies have shown that the effect of dopamine agents varies considerably, and can even go in opposite directions, depending on baseline levels of dopamine synthesis as measured with neurochemical PET (Cools and D'Esposito, 2011; Cools et al, 2009). Such variations could have been amplified by the use of a relatively low dose of sulpiride, which may act pre-synaptically to increase dopamine transmission in some individuals (Frank and O'Reilly, 2006). In the present study, it is therefore conceivable that the striatal response to near-misses was enhanced by sulpiride in some participants, but decreased in others, therefore nullifying any group effect. Note however that impulsiveness and working memory, as putative proxy measures of baseline dopamine levels, did not predict drug effects on near-miss responses (Supplementary Information). Alternatively, striatal responses to near-misses might not depend on the signaling from dopamine D2/D3 receptors, for which sulpiride has high affinity, but rather on D4 receptors for which sulpiride is 10 -fold less selective (Vallone et al, 2000). Supporting this idea, a recent study in rats has shown that selective D4 receptor agents were effective in modulating an analog of the near-miss effect, while selective D2/D3 receptors agents had no effect (Cocker et al, 2014).

One should also emphasize that the role of dopamine in pathological gambling has been hotly debated in recent years, due to the accumulation of inconsistent and null findings (Linnet, 2013; Potenza, 2013). For instance, pharmacological studies have shown that dopamine-enhancing and dopamine-blocking agents have intriguingly similar effects on gambling behavior in pathological gamblers (Zack and Poulos, 2004,2007). Furthermore, raclopride PET studies have failed to demonstrate, as is the case in substance addiction, that striatal dopamine D2 receptor density is altered in pathological gambling (Boileau et al, 2013; Clark et al, 2012). The complex and incomplete picture which is emerging suggests that other neurotransmitters are likely involved in gambling addiction. One interesting possibility in the case of near-misses is that they may operate through the noradrenergic system, via its role in computing 'risk prediction errors' (Preuschoff et al, 2011). Near-misses induce an upward re-evaluation of the winning probability, and thus elicit such risk prediction errors. Interestingly, pathological gamblers have been seen to display an underactive noradrenergic system (Pallanti et al, 2010), which could be driven by chronic activation in response to nearmiss events.

In short, our study reveals amplified striatal responses to near-miss events in pathological gamblers. This finding fits nicely with the idea that near-misses hijack the reward system and generate aberrant learning about skill acquisition, eventually leading to persistent and detrimental gambling behavior. These findings have implications in terms of prevention and treatment, such as further limiting the frequency of near-misses in gambling games (Harrigan, 2008), and incorporating more systematic demystification of illusion of control in cognitive behavioral therapy.

\section{FUNDING AND DISCLOSURE}

$\mathrm{RC}$ has been a consultant for Pfizer and Abbvie, but she is not an employee or a stock shareholder of either of these companies. LC is the Director of the Centre for Gambling Research at UBC, which is supported by the Province of British Columbia government and the British Columbia Lottery Corporation. LC has also consulted for Cambridge Cognition Ltd and has received a speaker honorarium from Svenska Spel (Sweden).

\section{ACKNOWLEDGMENTS}

GS was supported by a Veni grant from the Netherlands Research Organization (NWO).

\section{REFERENCES}

Balodis IM, Kober H, Worhunsky PD, Stevens MC, Pearlson GD, Potenza MN (2012). Diminished frontostriatal activity during processing of monetary rewards and losses in pathological gambling. Biol Psychiatry 71: 749-757.

Becker S, Gandhi W, Elfassy NM, Schweinhardt P (2013). The role of dopamine in the perceptual modulation of nociceptive stimuli by monetary wins or losses. Eur J Neurosci 38: 3080-3088.

Billieux J, Van der Linden M, Khazaal Y, Zullino D, Clark L (2012). Trait gambling cognitions predict near-miss experiences and persistence in laboratory slot machine gambling. Br J Psychol 103: 412-427.

Bischof A, Meyer C, Bischof G, John U, Wurst FM, Thon N et al (2015). Suicidal events among pathological gamblers: the role of comorbidity of axis I and axis II disorders. Psychiatry Res 225: 413-419.

Boileau I, Payer D, Chugani B, Lobo D, Behzadi A, Rusjan P et al (2013). The D2/3 dopamine receptor in pathological gambling: a positron emission tomography study with [11C]-(+)-propylhexahydro-naphtho-oxazin and [11C] raclopride. Addiction 108: 953.

Boileau I, Payer D, Chugani B, Lobo D, Houle S, Wilson A et al (2014). In vivo evidence for greater amphetamine-induced dopamine release in pathological gambling: a positron emission tomography study with [(11) C]-(+)-PHNO. Mol Psychiatry 19: 1305.

Chase HW, Clark L (2010). Gambling severity predicts midbrain response to near-miss outcomes. J Neurosci 30: 6180-6187.

Clark L (2010). Decision-making during gambling: an integration of cognitive and psychobiological approaches. Philos Trans $R$ Soc Lond B Biol Sci 365: 319-330.

Clark L, Lawrence AJ, Astley-Jones F, Gray N (2009). Gambling near-misses enhance motivation to gamble and recruit winrelated brain circuitry. Neuron 61: 481-490.

Clark L, Liu R, McKavanagh R, Garrett A, Dunn BD, Aitken MR (2013). Learning and affect following near-miss outcomes in simulated gambling. J Behav Decis Mak 26: 442-450.

Clark L, Stokes PR, Wu K, Michalczuk R, Benecke A, Watson BJ et al (2012). Striatal dopamine D 2/D 3 receptor binding in 
pathological gambling is correlated with mood-related impulsivity. Neuroimage 63: 40-46.

Clark L, Studer B, Bruss J, Tranel D, Bechara A (2014). Damage to insula abolishes cognitive distortions during simulated gambling. Proc Natl Acad Sci 111: 6098-6103.

Cocker PJ, Le Foll B, Rogers RD, Winstanley CA (2014). A selective role for dopamine $\mathrm{d} 4$ receptors in modulating reward expectancy in a Rodent Slot Machine Task. Biol Psychiatry 75: 817-824.

Cools R, Altamirano L, D’Esposito M (2006). Reversal learning in Parkinson's disease depends on medication status and outcome valence. Neuropsychologia 44: 1663-1673.

Cools R, D'Esposito M (2011). Inverted-U-shaped dopamine actions on human working memory and cognitive control. Biol Psychiatry 69: e113-e125.

Cools R, Frank MJ, Gibbs SE, Miyakawa A, Jagust W, D'Esposito M (2009). Striatal dopamine predicts outcome-specific reversal learning and its sensitivity to dopaminergic drug administration. J Neurosci 29: 1538-1543.

Côté D, Caron A, Aubert J, Desrochers V, Ladouceur R (2003). Near wins prolong gambling on a video lottery terminal. J Gambl Stud 19: 433-438.

de Ruiter MB, Veltman DJ, Goudriaan AE, Oosterlaan J, Sjoerds Z, van den Brink W (2009). Response perseveration and ventral prefrontal sensitivity to reward and punishment in male problem gamblers and smokers. Neuropsychopharmacology 34: 1027-1038.

Delgado MR (2007). Reward-related responses in the human striatum. Ann NY Acad Sci 1104: 70-88.

Dixon MJ, Harrigan KA, Jarick M, MacLaren V, Fugelsang JA, Sheepy E (2011). Psychophysiological arousal signatures of nearmisses in slot machine play. Int Gambl Stud 11: 393-407.

Dixon MJ, MacLaren V, Jarick M, Fugelsang JA, Harrigan KA (2013). The frustrating effects of just missing the jackpot: slot machine near-misses trigger large skin conductance responses, but no post-reinforcement pauses. J Gambl Stud 29: 661-674.

Eisenegger C, Naef M, Linssen A, Clark L, Gandamaneni PK, Müller U et al (2014). Role of dopamine D2 receptors in human reinforcement learning. Neuropsychopharmacology 39: 2366-2375.

Frank MJ, O’Reilly RC (2006). A mechanistic account of striatal dopamine function in human cognition: psychopharmacological studies with cabergoline and haloperidol. Behav Neurosci 120: 497.

Gläscher J (2009). Visualization of group inference data in functional neuroimaging. Neuroinformatics 7: 73-82.

Habib R, Dixon M (2010). Neurobehavioral evidence for the "NearMiss" effect in pathological gamblers. J Exp Anal Behav 93: 313-328.

Harrigan KA (2008). Slot machine structural characteristics: creating near misses using high award symbol ratios. Int J Ment Health Addict 6: 353-368.

Hewig J, Kretschmer N, Trippe RH, Hecht H, Coles MG, Holroyd CB et al (2010). Hypersensitivity to reward in problem gamblers. Biol Psychiatry 67: 781-783.

Janssen LK, Sescousse G, Hashemi MM, Timmer MHM, ter Huurne NP, Geurts DEM et al (2015). Abnormal modulation of reward versus punishment learning by a dopamine D2-receptor antagonist in pathological gamblers. Psychopharmacology 232: 3345-3353.

Jocham G, Klein TA, Ullsperger M (2014). Differential modulation of reinforcement learning by D2 dopamine and NMDA glutamate receptor antagonism. J Neurosci 34: 13151-13162.

Kassinove JI, Schare ML (2001). Effects of the "near miss" and the "big win" on persistence at slot machine gambling. Psychol Addict Behav 15: 155.

Lesieur HR, Blume SB (1987). The South Oaks Gambling Screen (SOGS): a new instrument for the identification of pathological gamblers. Am J Psychiatry 144: 1184-1188.

Leyton M, Vezina P (2012). On cue: striatal ups and downs in addictions. Biol Psychiatry 72: e21.
Leyton M, Vezina P (2013). Striatal ups and downs: their roles in vulnerability to addictions in humans. Neurosci Biobehav Rev 37: 1999-2014.

Linnet J (2013). The Iowa Gambling Task and the three fallacies of dopamine in gambling disorder. Front Psychol 4: 709.

Lorains FK, Cowlishaw S, Thomas SA (2011). Prevalence of comorbid disorders in problem and pathological gambling: systematic review and meta-analysis of population surveys. Addiction 106: 490-498.

MacLaren VV (2015). Video lottery is the most harmful form of gambling in Canada. J Gambl Stud 1-27 (e-pub ahead of print).

Maia TV, Frank MJ (2011). From reinforcement learning models to psychiatric and neurological disorders. Nat Neurosci 14: 154-162.

McCabe C, Huber A, Harmer CJ, Cowen PJ (2011). The D2 antagonist sulpiride modulates the neural processing of both rewarding and aversive stimuli in healthy volunteers. Psychopharmacology 217: 271-278.

Mehta MA, McGowan SW, Lawrence AD, Aitken MR, Montgomery AJ, Grasby PM (2003). Systemic sulpiride modulates striatal blood flow: relationships to spatial working memory and planning. Neuroimage 20: 1982-1994.

Mehta MA, Montgomery AJ, Kitamura Y, Grasby PM (2008). Dopamine D2 receptor occupancy levels of acute sulpiride challenges that produce working memory and learning impairments in healthy volunteers. Psychopharmacology 196: 157-165.

Michalczuk R, Bowden-Jones H, Verdejo-Garcia A, Clark L (2011). Impulsivity and cognitive distortions in pathological gamblers attending the UK National Problem Gambling Clinic: a preliminary report. Psychol Med 41: 2625-2635.

Morgado P, Marques F, Silva MB, Sousa N, Cerqueira JJ (2014). A novel risk-based decision-making paradigm. Front Behav Neurosci 8: 45.

Nowak DE, Aloe AM (2014). The prevalence of pathological gambling among college students: a meta-analytic synthesis, 2005-2013. J Gambl Stud 30: 819-843.

Oberg SA, Christie GJ, Tata MS (2011). Problem gamblers exhibit reward hypersensitivity in medial frontal cortex during gambling. Neuropsychologia 49: 3768-3775.

Oswald LM, Wand GS, Wong DF, Brown CH, Kuwabara H, Brašić JR (2015). Risky decision-making and ventral striatal dopamine responses to amphetamine: a positron emission tomography [11 C] raclopride study in healthy adults. Neuroimage 113: 26-36.

Pallanti S, Bernardi S, Allen A, Chaplin W, Watner D, DeCaria C et al (2010). Noradrenergic function in pathological gambling: blunted growth hormone response to clonidine. J Psychopharmacol 24: 847-853.

Pessiglione M, Seymour B, Flandin G, Dolan RJ, Frith CD (2006). Dopamine-dependent prediction errors underpin reward-seeking behaviour in humans. Nature 442: 1042-1045.

Porchet RI, Boekhoudt L, Studer B, Gandamaneni PK, Rani N, Binnamangala S et al (2013). Opioidergic and dopaminergic manipulation of gambling tendencies: a preliminary study in male recreational gamblers. Front Behav Neurosci 77: 138.

Potenza MN (2013). How central is dopamine to pathological gambling or gambling disorder? Front Behav Neurosci 7: 206.

Preuschoff K, Marius't Hart B, Einhäuser W (2011). Pupil dilation signals surprise: evidence for noradrenaline's role in decision making. Front Neurosci 5: 115.

Reuter J, Raedler T, Rose M, Hand I, Glascher J, Buchel C (2005). Pathological gambling is linked to reduced activation of the mesolimbic reward system. Nat Neurosci 8: 147-148.

Riba J, Krämer UM, Heldmann M, Richter S, Münte TF (2008). Dopamine agonist increases risk taking but blunts reward-related brain activity. PLoS One 3: e2479-e2479.

Sescousse G, Barbalat G, Domenech P, Dreher JC (2013). Imbalance in the sensitivity to different types of rewards in pathological gambling. Brain 136: 2527-2538. 
Shao R, Read J, Behrens T, Rogers R (2013). Shifts in reinforcement signalling while playing slot-machines as a function of prior experience and impulsivity. Transl Psychiatry 3: e213.

Sheehan DV, Lecrubier Y, Sheehan KH, Amorim P, Janavs J, Weiller E et al (1998). The Mini-International Neuropsychiatric Interview (MINI): the development and validation of a structured diagnostic psychiatric interview for DSM-IV and ICD-10. J Clin Psychiatry 59: 22-33.

St Onge JR, Floresco SB (2009). Dopaminergic modulation of riskbased decision making. Neuropsychopharmacology 34: 681-697.

Stange M, Graydon C, Dixon MJ (2015). 'I was that close': investigating players' reactions to losses, wins, and near-misses on scratch cards. J Gambl Stud 32: 187-203.

Steeves T, Miyasaki J, Zurowski M, Lang A, Pellecchia G, Van Eimeren T et al (2009). Increased striatal dopamine release in Parkinsonian patients with pathological gambling: a [11C] raclopride PET study. Brain 132: 1376-1385.

Vallone D, Picetti R, Borrelli E (2000). Structure and function of dopamine receptors. Neurosci Biobehav Rev 24: 125-132.

van der Schaaf ME, van Schouwenburg MR, Geurts DE, Schellekens AF, Buitelaar JK, Verkes RJ et al (2014). Establishing the dopamine dependency of human striatal signals during reward and punishment reversal learning. Cereb Cortex 24: 633-642.

Verhagen L, Dijkerman HC, Grol MJ, Toni I (2008). Perceptuomotor interactions during prehension movements. J Neurosci 28: $4726-4735$
Wadhwa M, Kim JC (2015). Can a Near Win Kindle Motivation? The impact of nearly winning on motivation for unrelated rewards. Psychol Sci 26: 701-708.

Winstanley CA, Cocker PJ, Rogers RD (2011). Dopamine modulates reward expectancy during performance of a slot machine task in rats: evidence for a 'near-miss' effect. Neuropsychopharmacology 36: 913-925.

Worhunsky PD, Malison RT, Rogers RD, Potenza MN (2014). Altered neural correlates of reward and loss processing during simulated slot-machine fMRI in pathological gambling and cocaine dependence. Drug Alcohol Depend 145: 77-86.

Wu Y, Dijk E, Clark L (2015). Near-wins and near-losses in gambling: a behavioral and facial EMG study. Psychophysiology 52: 359-366.

Xue G, He Q, Lu Z-L, Levin IP, Dong Q, Bechara A (2013). Agency modulates the lateral and medial prefrontal cortex responses in belief-based decision making. PLoS One 8: e65274.

Xue G, Juan C-H, Chang C-F, Lu Z-L, Dong Q (2012). Lateral prefrontal cortex contributes to maladaptive decisions. Proc Natl Acad Sci 109: 4401-4406.

Zack M, Poulos C (2004). Amphetamine primes motivation to gamble and gambling-related semantic networks in problem gamblers. Neuropsychopharmacology 29: 195-207.

Zack M, Poulos CX (2007). A D2 antagonist enhances the rewarding and priming effects of a gambling episode in pathological gamblers. Neuropsychopharmacology 32: 1678-1686.

Supplementary Information accompanies the paper on the Neuropsychopharmacology website (http://www.nature.com/npp) 